\title{
Against Mathematical Convenientism
}

\begin{abstract}
Indispensablists argue that when our belief system conflicts with our experiences, we can negate a mathematical belief but we do not because if we do, we would have to make an excessive revision of our belief system. Thus, we retain a mathematical belief not because we have good evidence for it but because it is convenient to do so. I call this view 'mathematical convenientism.' I argue that mathematical convenientism commits the consequential fallacy and that it demolishes the Quine-Putnam indispensability argument and Baker's enhanced indispensability argument.
\end{abstract}

\section{Keywords}

Mathematical Confirmationism, Mathematical Convenientism, Indispensability Argument, Mathematical Realism

Seungbae Park

Division of General Studies

Ulsan National Institute of Science and Technology

nature@unist.ac.kr

Park, Seungbae (2016). “Against Mathematical Convenientism,” Axiomathes 26 (2): 115-122.

\section{Introduction}

No pure mathematical sentence like $1+1=2$ was refuted in the history of science. Charles Parsons (1983) argues that the historical fact undermines the Quine-Putnam indispensability argument according to which observations confirm mathematical beliefs as well as concrete beliefs, beliefs that are about concrete objects like trees and electrons. Indispensablists reply that we can negate a mathematical belief but we do not because negating a mathematical belief has the unpalatable consequence of having to make an excessive revision of our belief system. Thus, we retain a mathematical belief not because we have good evidence for it but because it is convenient to do so. I call this view 'mathematical convenientism.'

This paper aims to show that it is incoherent for indispensablists to rely on mathematical convenientism to meet Parsons's objection. I proceed as follows. In Section 2, I explicate the Quine-Putnam indispensability argument, the target of Parsons's criticism. I expose two important ideas that are inherent in the Quine-Putnam indispensability argument. They are mathematical realism and mathematical confirmationism. Mathematical realism is the view that mathematical objects are real, and mathematical confirmationism is the view that observations can confirm a mathematical belief. In Section 3, I argue that mathematical convenientism commits the consequential fallacy and that it demolishes both mathematical realism and confirmationism. In the end, it will become clear that indispensablists cannot avail themselves of mathematical convenientism.

\section{The Quine-Putnam Indispensability Argument}

Mathematical realism holds that mathematical objects, such as numbers, triangles, and functions, are real. Where do they exist? Mathematical realism asserts that they exist not in the concrete world but in the abstract world. The concrete world is the world in which there are concrete objects, such as human beings, stones, and electrons. They are spatial, temporal, and causal objects. They can causally interact with one another. In contrast, the abstract world is the world in which there are abstract objects, such as mathematical objects and 
propositions. They are aspatial, atemporal, and non-causal objects. There is no space and no flow of time in the abstract world. Hence, abstract objects can causally interact neither with one another nor with concrete objects.

Why should we believe that mathematical objects exist in the abstract world? A famous argument advanced in support of mathematical realism is the Quine-Putnam indispensability argument. It is defended by Willard V. O. Quine (1948, 1980, 1992), Hilary Putnam (1971), Michael Resnik (1997), and Mark Colyvan (2001). It says roughly that mathematics is indispensable to our best scientific theories, observations confirm mathematical components as well as concrete components of our best scientific theories, and hence we ought to believe that mathematical entities are real, just as we ought to believe that theoretical entities, such as electrons and black holes, are real. On the indispensablist account, we ought to believe that all and only objects postulated by our best scientific theories. Thus, we ought not to believe, for example, that witches are real because no scientific theory posits their existence. But we ought to believe that mathematical and theoretical entities are real because our best scientific theories posit their existence. Quine (1980: 45), Putnam (1979: 347), and Colyvan (2006: 226-227) claim that it is intellectually dishonest to believe that theoretical entities are real but that mathematical entities are not. Keep in mind that as far as these eminent philosophers of mathematics are concerned, mathematical entities are epistemically on a par with theoretical entities.

The Quine-Putnam indispensability argument contains two ideas that are important for the purpose of this paper. The first idea is the view that mathematical objects are real, and the second idea is the view that observations confer justification on a mathematical sentence. A mathematical hypothesis can be confirmed by empirical data, just as a concrete hypothesis can be confirmed by empirical data. I earlier named this philosophical view on confirmation 'mathematical confirmationism.' Indispensablists use mathematical confirmationism as a means to arrive at mathematical realism. In other words, mathematical confirmationism is indispensablists' answer to the epistemological question: How do you know that mathematical objects exist?

Both mathematical realism and mathematical confirmationism presuppose that a mathematical statement is not analytic but synthetic. An analytic statement is true or false solely in virtue of the meaning of the statement, whereas a synthetic statement is true or false in virtue of the meaning of the statement and in virtue of the way the world is. An analytic statement does not contain information about the world, whereas a synthetic statement does. So if a mathematical statement is analytic, mathematical realism collapses. The mathematical statement, ' $1+1=2$,' if analytically true, does not entail that numbers are real. Also, an analytic statement is not the kind of statement that can be confirmed or disconfirmed by observations, whereas a synthetic statement is the kind of statement that can be confirmed or disconfirmed by observations. So if a mathematical statement is analytic, the justification for it does not come from observations, i.e., mathematical confirmationism is false. Keep in mind that an analytic statement is not subject to an empirical refutation, whereas a synthetic statement is subject to an empirical refutation.

\section{Mathematical Convenientism}

Many objections have been raised against the Quine-Putnam indispensability in the literature. This paper concerns only Parsons's objection (1983) that if a mathematical sentence can be confirmed by observations, it should also be able to be disconfirmed by observations. But "no proposition of pure mathematics has been falsified" (Parsons, 1983: 196). Why is it, for example, that ' $1+1=2$ ' was not refuted in the history of science? The history of science constitutes the prima facie reason for thinking that a mathematical sentence is not the kind of 
sentence that can be confirmed or disconfirmed by empirical data. It appears that a mathematical sentence is not synthetic but analytic, contrary to what the Quine-Putnam indispensability argument presupposes.

In my view, indispensablists should take Parsons's objection seriously because they contend, as we noted before, that it is intellectually dishonest to believe that theoretical entities are real, but mathematical entities are not. A similar attack can be directed at indispensablists, viz., a double standard is involved in the suggestion that a concrete hypothesis is subject to an empirical refutation, but a mathematical hypothesis is not. Under the indispensablist framework, it is intellectually dishonest to believe that a concrete hypothesis can, but a mathematical hypothesis cannot, be disconfirmed by observations.

Moreover, Parsons's observation of the history of science can be strengthened. There is a gross historical asymmetry between theoretical and mathematical entities. Many theoretical entities like the celestial sphere, ether, phlogiston, and caloric fluids turned out to be nonexistent, as Larry Laudan's list (1981: 33) of past theories indicates. Laudan's list is often cited in the philosophy of science literature as supporting the pessimistic induction that since past theories turned out to be false, present theories will also turn out be false. Laudan's list, however, does not show that mathematical objects like numbers, triangles, and functions turned out to be nonexistent. Thus, the history of science indicates that a mathematical statement is not the kind of statement which can be confirmed or disconfirmed by observations.

How do indispensablists respond to Parsons's objection in the literature? Quine replies that we can, but we do not, negate a mathematical sentence, "for mathematics infiltrates all branches of our system of the world, and its disruption would reverberate intolerably" (Quine, 1992: 15). In other words, negating a concrete belief requires a small revision of our belief system, but negating a mathematical belief requires an exorbitant revision of our belief system. Hence, we ought to displace a concrete belief with a new one rather than a mathematical belief with a new one. Colyvan concurs with Quine, saying that "it's not that mathematical theories are unfalsifiable in any absolute sense; it's just that whenever they are part of a falsified package, the mathematical portion is unusually salvaged for reasons of minimum mutilation to the web of belief" (2001: 126). Resnik agrees with Quine and Colyvan, saying that modifying a mathematical theory "is likely to send reverberations into currently quiescent areas of science" (Resnik, 1997: 125-126). Notice that according to these philosophers, the reason for safeguarding a mathematical belief is not epistemic but pragmatic. To put it another way, we adhere to a mathematical belief not because we have sufficient evidence revealing it to be true but because we want to avoid the inconvenience of revising inordinately many concrete beliefs.

An interesting consequence follows from the preceding indispensablist account of why we do not discard a mathematical belief. Indispensablists are mathematical realists believing that mathematical objects are real. They now claim, however, that we retain a mathematical belief not because we have the good evidence for it but because it is convenient to do so. I earlier named this view 'mathematical convenientism.' Mathematical convenientism is motivated by a pragmatic consideration as opposed to an epistemic consideration. Therefore, mathematical convenientism commits the consequential fallacy.

The consequential fallacy occurs when we conflate the effect of holding a belief to be true with the evidence for it. Specifically, we hold a belief to be true not because we have good evidence for it but because it is useful. To use Merrilee Salmon's example (2007: 210), some smokers believe that heavy smoking is not harmful to our health on the grounds that they are happier if they believe so. To use a famous example from philosophy of religion, Blaise Pascal (1623-1662) argued that we ought to believe in God not because we have the 
sufficient evidence for his existence but because the belief in him might be tremendously beneficial to us after we die. Pascal provided the pragmatic justification as opposed to the epistemic justification for the belief that God exists. Both the smokers and Pascal committed the consequential fallacy.

Analogously, indispensablists commit the consequential fallacy when they attempt to justify their choice of one belief system over another by appealing to the pragmatic consideration that discarding a mathematical belief involves an excessive change in our preexisting belief system. There are two rival belief systems, $S_{1}$ and $S_{2} . S_{1}$ is the result of discarding a concrete belief in our current belief system. $S_{2}$ is the result of discarding a mathematical belief in our current belief system. Both $S_{1}$ and $S_{2}$ can cope with our experiences. Indispensablists claim that we should choose $S_{1}$ over $S_{2}$ because it is convenient to do so. Thus, we safeguard a mathematical belief not because we have good evidence for it but because if we do so, we do not have to go through the pain of revising numerous concrete beliefs. Indispensablists have confounded the effect of retaining a mathematical belief with the evidence for it.

Let me elucidate three disastrous consequences of mathematical convenientism on indispensablists. First, mathematical convenientism wreaks havoc on mathematical beliefs. If indispensablists choose $S_{1}$ over $S_{2}$ on the grounds that it is convenient to do so, their mathematical beliefs are not epistemically justified but practically justified. They believe, for example, that $1+1=2$ not because they have good evidence for it but because they are happier if they do so. It follows that indispensablists can no longer believe that mathematical objects are real. Thus, mathematical realism collapses.

Second, mathematical convenientism also wreaks havoc on observational beliefs. Indispensablists believe, for example, that a cat is on the mat after they have chosen $\mathrm{S}_{1}$ over $\mathrm{S}_{2}$. Mathematical convenientism, however, implies that their observational beliefs are not epistemically warranted but practically warranted. Indispensablists believe, for example, that a cat is on the mat not because they have good evidence for it but because it is convenient to do so. It follows that indispensablists can no longer say that observational beliefs support a mathematical hypothesis. After all, an epistemically unjustified belief cannot confer epistemic justification on another belief. Thus, mathematical confirmationism collapses too.

Third, mathematical convenientism destroys not only the Quine-Putnam indispensability argument but also the enhanced indispensability argument recently defended by Alan Baker $(2005,2009,2012)$. Baker's enhanced indispensability argument holds that a mathematical hypothesis can best explain concrete phenomena, so if we believe that a concrete hypothesis is true on the grounds that it best explains concrete phenomena, we should also believe that a mathematical hypothesis is true. Baker provides the famous example to justify his contention that a mathematical hypothesis can best explain concrete phenomena. He does not claim that a mathematical hypothesis is confirmed by observations because it is an indispensable part of our scientific theory which is confirmed by observations. He rather claims that like a concrete hypothesis, a mathematical hypothesis can be directly supported by observations.

Baker's enhanced indispensability argument is better than the Quine-Putnam indispensability argument because it is not committed to the problematic doctrine, confirmational holism. Confirmational holism holds that observations confirm not just some parts of a scientific theory but all parts of a scientific theory. Confirmational holism has received severe criticisms from Penelope Maddy (1992) and Jacob Busch (2012). Maddy observes that we do not believe that ideal entities, such as frictionless slope and continuous fluids, are real, although they are indispensable parts of our best scientific theories. Busch appeals to Philip Kitcher (1993: 140-149) and Stathis Psillos (1999, Chapters 5 and 6) who 
distinguish between working components and idle components of the ether theory and the caloric theory, respectively, and then argue that empirical evidence supported working components, but not idle components of the past theories. It is no longer clear these days whether there is any indispensablist who sticks to confirmational holism.

Baker's enhanced indispensability argument is similar to the Quine-Putnam indispensability argument in an important respect. They are both committed to mathematical realism and confirmationism. So the enhanced indispensability argument is also subject to Parsons's objection, and if Baker appeals to mathematical convenientism to confront Parsons's objection, his enhanced indispensability argument, although better than the QuinePutnam indispensability argument, also falls preys to my criticism that mathematical convenientism destroys both mathematical realism and confirmationism.

\section{Objection and Reply}

A referee brings my attention to the recent attempts (Lakoff and Núñez, 2001; Dehaene, 2011) in cognitive science to give an evolutionary account of mathematics. On the evolutionary account, our minds are genetically hardwired to think that $1+1=2$ because of the environment in which our ancestors evolved. Our minds would be genetically hardwired to think that $1+1=1$, if our ancestors had evolved in a different environment:

At our scale, the world is mostly made up of separable objects that combine into sets according to the familiar equation $1+1=2$. This is why evolution has anchored this rule in our genes. Perhaps our arithmetic would have been radically different if, like cherubs, we had evolved in the heavens where one cloud plus another cloud was still one cloud. (Dehaene, 2011: 231)

The evolutionary account of mathematics has an interesting implication on my previous contention concerning the history of science. In Section 3, I said that the history of science gives rise to the suspicion that mathematical sentences like $1+1=2$ are analytic, so indispensablists have the burden to explain why no pure mathematical sentence was refuted in the history of science. This analytic account can be undermined by the evolutionary account that no pure mathematical sentence was discarded in the history of science because pure mathematical sentences are genetically hardwired in our minds. It was psychologically impossible for our scientific predecessors to get the belief $1+1=2$ off their minds.

The referee's objection is reasonable. The evolutionary account indeed undermines the analytic account. It is not clear, however, that the evolutionary account helps indispensablists. Mathematical realism collapses, if it is true that we think that $1+1=2$ not because we have good evidence for it but because we are evolutionarily hardwired to think so. Mathematical realists confront a vexing question: Why do we believe that $1+1=2$ as opposed to $1+1=1$ ? They cannot say that we believe so because our ancestors evolved on the earth as opposed to on the heaven and because the belief $1+1=2$ was useful on the earth, although the belief $1+1=1$ was useful on the heaven. To say so is to commit the consequential fallacy. Hence, indispensablists cannot appeal to the evolutionary account to meet Parsons's challenge.

\section{Conclusion}

Indispensablists appeal to mathematical convenientism to blunt Parsons's objection that no sentence of pure mathematics was refuted in the history of science. Mathematical convenientism holds that we can but do not negate a mathematical belief because negating a concrete belief requires a minor revision whereas negating a mathematical belief requires a sweeping revision of our belief system. I objected that on a close examination, mathematical convenientism demolishes both mathematical realism and convenientism. The spirit of the 
Quine-Putnam indispensability argument and Baker's enhanced indispensability argument is that observations confer epistemic justification on our mathematical beliefs. Indispensablists, however, unwittingly have gone against the spirit in the attempt to defend mathematical realism from Parsons's challenge.

Indispensablists are in a dilemma. A mathematical sentence is either susceptible or immune to an empirical refutation. On the one hand, if they say that it is susceptible to an empirical refutation, they are free from the charge that they hold a double standard with respect to mathematical and concrete sentences, and they can adhere to mathematical confirmationism. They, however, have the burden to explain why no sentence of pure mathematics was disconfirmed in the history of science, and why theoretical entities like ether and phlogiston were thrown out, but mathematical entities like numbers and triangles were not. On the other hand, if indispensablists say that a mathematical sentence is immune to an empirical refutation, they do not have the burden to explain why $1+1=2$ was not refuted in the history of science and why there is the gross historical asymmetry between theoretical and mathematical entities. This position, however, invites the suspicion that mathematical sentences are not synthetic but analytic. The conclusion of this paper is that it is better for indispensablists to be in this dilemma than to escape from it with the use of mathematical convenientism.

\section{References}

Baker, Alan (2005). "Are There Genuine Mathematical Explanations of Physical Phenomena?", Mind 114 (454): 223-237.

(2009). "Mathematical Explanation in Science", British Journal for the Philosophy of Science 60 (3): 611-633.

(2012). “Science-Driven Mathematical Explanation”, Mind 121 (482): 243-267.

Busch, Jacob (2012). "The Indispensability Argument for Mathematical Realism and Scientific Realism”, Journal for General Philosophy of Science 43 (1): 3-9.

Colyvan, Mark (2001). The Indispensability of Mathematics. New York: Oxford University Press.

(2006). "Scientific Realism and Mathematical Nominalism: A Marriage Made in Hell", In Colin Cheyne and John Worrall (eds.), Rationality and Reality: Conversations with Alan Musgrave. Dordrecht, The Netherlands: Springer.

Deheane, Stanislas (2011). The Number Sense: How the Mind Creates Mathematics. Oxford: Oxford University Press.

Kitcher, Philip (1993). The Advancement of Science: Science without Legend Objectivity without Illusion. New York: Oxford University Press.

Lakoff, George and Rafael E. Núñez (2001). Where Mathematics Comes from: How the Embodied Mind Brings Mathematics into Being. New York: Basic Books.

Laudan, Larry (1981). “A Confutation of Convergent Realism”, Philosophy of Science 48 (1): $19-49$. 
Maddy, Penelope (1992). "Indispensability and Practice", The Journal of Philosophy 89 (6): 275-289.

Parsons, Charles (1983). Philosophy in Mathematics: Selected Essays. Ithaca, New York: Cornell University Press.

Psillos, Stathis (1999). Scientific Realism: How Science Tracks Truth. New York: Routledge.

Putnam, Hilary (1971). Philosophy of Logic. New York: Harper and Row.

(1979). "Philosophy of Logic", Reprinted in Mathematics Matter and Method: Philosophical Papers. Vol. 1, $2^{\text {nd }}$ ed., Cambridge: Cambridge University Press.

Quine, Willard V. O. (1948) “On What There Is”, Review of Metaphysics 2 (5): 21-38.

-------- (1980). From a Logical Point of View. $2^{\text {nd }}$ ed., Cambridge, MA: Harvard University Press.

(1992). Pursuit of Truth. Cambridge MA: Harvard University Press.

Resnik, Michael (1997). Mathematics as a Science of Patterns. Oxford: Clarendon Press.

Salmon, Merrilee (2007). Introduction to Logic and Critical Thinking. 5th ed., US: Thomson Wadsworth. 